\title{
Philosophiques
}

\section{Husserl à Halle (1886-1901)}

\section{Denis Fisette}

Volume 36, numéro 2, automne 2009

\section{Edmund Husserl (1859-1938)}

URI : https://id.erudit.org/iderudit/039474ar

DOI : https://doi.org/10.7202/039474ar

Aller au sommaire du numéro

\section{Éditeur(s)}

Société de philosophie du Québec

ISSN

0316-2923 (imprimé)

1492-1391 (numérique)

Découvrir la revue

\section{Citer cet article}

Fisette, D. (2009). Husserl à Halle (1886-1901). Philosophiques, 36(2), 277-306. https://doi.org/10.7202/039474ar

\section{Résumé de l'article}

Cette présentation générale a pour but d'expliciter l'arrière-plan théorique et historique des études réunies dans ce numéro de Philosophiques, lesquelles portent principalement sur l'oeuvre de Husserl durant la période de Halle (1886-1901). Après une description succincte des années d'apprentissage de Husserl en philosophie entre 1876 et ses études avec Brentano à Vienne, je décris les étapes qui ont marqué le développement de sa philosophie de son arrivée à Halle en 1886 jusqu'à la publication des Recherches logiques (1900-1901) : ses études sous la direction de C. Stumpf, la publication du premier volume de sa Philosophie de l'arithmétique, les recherches relatives au projet de la rédaction du deuxième volume de cet ouvrage, l'abandon de ce projet et l'élaboration de la phénoménologie des Recherches logiques. La dernière partie est un examen sommaire des enjeux philosophiques sous-jacents à la disputatio autour d'un ouvrage récent sur Husserl.
Ce document est protégé par la loi sur le droit d'auteur. L'utilisation des services d'Érudit (y compris la reproduction) est assujettie à sa politique d'utilisation que vous pouvez consulter en ligne.

https://apropos.erudit.org/fr/usagers/politique-dutilisation/ 


\title{
Husserl à Halle (1886-1901)*
}

\author{
DENIS FISETTE
}

Université du Québec à Montréal

\begin{abstract}
RÉSUMÉ. - Cette présentation générale a pour but d'expliciter l'arrière-plan théorique et historique des études réunies dans ce numéro de Philosophiques, lesquelles portent principalement sur l'œuvre de Husserl durant la période de Halle (1886-1901). Après une description succincte des années d'apprentissage de Husserl en philosophie entre 1876 et ses études avec Brentano à Vienne, je décris les étapes qui ont marqué le développement de sa philosophie de son arrivée à Halle en 1886 jusqu'à la publication des Recherches logiques (19001901): ses études sous la direction de C. Stumpf, la publication du premier volume de sa Philosophie de l'arithmétique, les recherches relatives au projet de la rédaction du deuxième volume de cet ouvrage, l'abandon de ce projet et l'élaboration de la phénoménologie des Recherches logiques. La dernière partie est un examen sommaire des enjeux philosophiques sous-jacents à la disputatio autour d'un ouvrage récent sur Husserl.
\end{abstract}

\begin{abstract}
This presentation aims to clarify the historical and theoretical background of the studies included in this issue of Philosophiques, which focus on the work of Husserl during the period of Halle (1886-1901). After a brief description of Husserl's early years of apprenticeship in philosophy between 1876 and his studies with Brentano in Vienna, I identify several steps that marked the development of his philosophy from his arrival in Halle (1886) to the publication of the Logical Investigations (1900-1901): his studies under the direction of C. Stumpf, the publication of the first volume of Philosophy of Arithmetic, the researches belonging to the project of the second volume of this work, the abandonment of this project and the development of the phenomenology of the Logical Investigations. The last part of this paper is an examination of the philosophical issues underlying the disputatio on D. W. Smith's recent book on Husserl's philosophy.
\end{abstract}

Au tout début des années 1930, Husserl reçoit une lettre de V. K. Skrach, alors secrétaire du président de la République tchécoslovaque, l'invitant à contribuer à un Festschrift consacré à son compatriote morave Thomas G. Masaryk à l'occasion de son $80^{\mathrm{e}}$ anniversaire de naissance ${ }^{1}$. Husserl rédige sur le champ un manuscrit intitulé "De l'origine $»^{2}$ où il traite de la place de la psychologie intentionnelle dans sa phénoménologie et du thème de l'élucidation de l'origine des idées. En choisissant ce thème typiquement

* Je remercie le Conseil de recherches en sciences humaines du Canada pour son support financier et G. Fréchette pour ses remarques pertinentes sur cette présentation.

1. Lettre de Husserl à Vasil K. Skrach datée du 31 janvier 1930, in Husserl, Briefwechsel, Bd. I, p. 117. J'utilise l'abréviation Hua suivie du numéro du volume et de la pagination en référence aux œuvres complètes de Husserl (Husserliana).

2. Husserl, «Über Ursprung. Die reine Psychologie und die Aufgabe einer Ursprungsklärung unserer Ideen », Hua XXVII, p. 129-163. 
brentanien, Husserl voulait sans doute commémorer les origines brentaniennes de sa propre philosophie, ainsi que celle de Masaryk, et faire le point sur la question du rapport de ses positions philosophiques au début des années 1930 avec la philosophie de Brentano. C'est ce que confirment certaines remarques de ce texte, qui n'a cependant jamais été achevé3. Cinq années plus tard, Husserl est invité à prononcer une conférence devant le Cercle linguistique de Prague et il fait part à Masaryk de son souhait de le revoir bientôt dans sa patrie 4 . Les 14 et 15 novembre 1935, il prononce en effet une conférence intitulée «La psychologie dans la crise des sciences européennes ${ }^{5}$ », dans laquelle il reprend un thème très proche de celui qu'il destinait au Festschrift de Masaryk. Dans cet environnement de Prague, qui était encore à cette époque un bastion des études brentaniennes, Husserl décrit sa relation à la psychologie descriptive de Brentano dans les termes suivants :

Parmi les réactions contre la psychologie physicaliste et physiologique [...], la tentative énergique et géniale de Brentano de réformer la psychologie avait une signification particulière, celle de promouvoir une psychologie reposant véritablement sur l'expérience interne et ainsi sur le fondement d'une analyse purement descriptive de l'intériorité psychique. L'introduction du concept scolastique d'intentionnalité en tant que trait caractéristique essentiel du psychique fut tout simplement révolutionnaire. [...] Et pourtant, la réforme psychologique de Brentano ne put s'accomplir et ne suffit pas à instituer une véritable psychologie intentionnelle. En matière de science, il n'y a pas de dissimulation [Verschweigen]. Et je dois donc expliquer ici pourquoi, malgré toute mon estime personnelle à l'endroit de mon maître, laquelle rendit si difficile pour moi de séparer mon chemin du sien, je ne pus méthodologiquement et objectivement continuer sa psychologie ${ }^{6}$.

En reconnaissant sa dette à l'endroit de Brentano et en choisissant le thème de l'origine afin de commémorer l'anniversaire de Masaryk, Husserl voulait aussi rappeler l'influence déterminante que celui-ci avait exercée près de cinquante années plus tôt sur le cours de sa vie en le convainquant d'entreprendre des études en philosophie auprès du maître ${ }^{7}$. Puisque cet épisode de la vie intellectuelle de Husserl est peu connu même dans les études

3. Husserl, Hua XXVII, p. 144.

4. Lettre de Husserl à Masaryk datée du 3 janvier 1935: «J'apprends que dans le Cercle linguistique de Prague nouvellement fondé, on a l'intention de m'inviter aux séminaires. Malgré mon âge et avec la permission officielle que cela présuppose, je viendrai volontiers dans la perspective réjouissante de pouvoir éventuellement vous voir. C'est d'ailleurs une joie de ma vieillesse que d'apprendre finalement et sur le tard que mon travail de toute une vie suscite quelque intérêt dans ma patrie" (Husserl, Briefwechsel, Bd. I, p. 119).

5. Husserl, «Die Psychologie in der Krise der europaischen Wissenschaft», in Hua XXIX, p. 103-139.

6. Husserl, Hua XXIX, p. 123-124.

7. Cf. K. Schuhmann, «Husserl and Masaryk». 
husserliennes et qu'il est déterminant pour bien comprendre l'arrière-plan philosophique de ce jeune mathématicien de formation, je voudrais le décrire schématiquement en guise d'introduction à la description que je ferai ensuite de la période de Halle.

Depuis ses premières années d'étude à Leipzig, à l'âge de 17 ans, jusqu'à la publication du premier volume de Philosophie de l'arithmétique, les années d'apprentissage du jeune Husserl ont été marquées par la philosophie de Brentano, qui représente le cœur de sa formation philosophique. En 1876, Husserl se rendit à Leipzig pour y étudier l'astronomie, les mathématiques et la philosophie, et y demeura pendant trois semestres. C'est durant cette période qu'il fit la rencontre de Masaryk, lequel revenait tout juste d'un séjour d'étude à Vienne où il avait soutenu, sous la direction de Brentano, une thèse de doctorat sur Platon ${ }^{9}$. Cette rencontre eut lieu dans un cours de philosophie, vraisemblablement donné par W. Wundt, déjà connu à cette époque ${ }^{10}$, que Husserl fréquentait uniquement par curiosité et pour sa formation personnelle. Comme l'explique Husserl dans une lettre à caractère autobiographique adressée au secrétaire de Masaryk:

J'arrivai à Leipzig à l'âge de 17 ans et demi afin d'étudier l'astronomie. Au même moment (à l'automne de 1876) arriva aussi Masaryk, un jeune docteur en philosophie qui était alors éducateur du fils d'un banquier. Comme nous étions compatriotes moraves, nous fîmes rapidement connaissance, et Masaryk devint pour moi un mentor et ami. Conjointement avec lui, je fréquentais des leçons de philosophie - pour ma formation générale à cette époque, et non comme orientation - et puisqu'il était docteur en philosophie et naturellement beaucoup plus avancé que moi, il m'aida à comprendre la matière et montra au profane que j'étais les voies de la libre pensée ${ }^{11}$.

En août 1877, Masaryk retourne à Vienne pour y préparer sa thèse d'habilitation sous la direction de Brentano. Il incite Husserl à l'accompagner

8. Husserl, Philosophie de l'arithmétique.

9. On sait que Masaryk a assisté aux leçons de Brentano de 1874 à 1876, notamment à celles sur les textes philosophiques d'Aristote, la philosophie pratique et la philosophie chrétienne en rapport avec Hume. En mars 1876, il soutient une thèse intitulée Das Wesen der Seele bei Plato. Eine kritische Studie vom empirischen Standpunkt, thèse qui n'a jamais été publiee (cf. K. Capek, Gespräche mit Masaryk, p 71). Brentano commente la thèse de son étudiant de la manière suivante: "[C]ette dissertation est indiscutablement rédigée avec amour et soin.» Masaryk possède "une connaissance approfondie de l'intégralité des œuvres de Platon [...]. L'auteur a manifesté sa capacité pour la recherche dans le domaine de l'histoire de la philosophie, mais aussi, dans une certaine mesure, une capacité de réflexion spéculative personnelle sur ce problème » (passages cités in D. Fisette et G. Fréchette «Le legs de Brentano », p. 31).

10. Cf. la lettre à Brentano datée du 23 novembre 1876 dans laquelle Masaryk décrit brièvement l'enseignement de Wundt et de Drobisch, et sa rencontre avec l'empiriocriticiste R. Avenarius.

11. Lettre du 21-08-1936 à F. Jancik, Husserl, Briefwechsel, Bd. VIII, p. 58-59. 
afin de faire la connaissance de Brentano ${ }^{12}$, mais Husserl demeure à Leipzig jusqu'à la fin du semestre d'hiver 1877-1878 et décide alors de se consacrer à l'étude des mathématiques à Berlin, où se trouvent alors les célèbres mathématiciens Kronecker et Weierstraß. Il continue néanmoins de s'intéresser à la philosophie et assiste aux leçons de Friedrich Paulsen à Berlin. Husserl demeure à Berlin jusqu'au semestre d'hiver de 1880-1881 et décide finalement de retourner à Vienne parce qu'il estime que, en tant que Juif de nationalité autrichienne, les probabilités d'entreprendre avec succès une carrière académique en Autriche sont plus grandes qu'en Allemagne ${ }^{13}$. Il retourne donc à Vienne en mars 1881 afin de préparer sa dissertation avec Leo Königsberger, un ancien étudiant de Weierstraß, et retrouve son ami Masaryk, qui était depuis 1879 privatdozent à Vienne. Husserl entretient alors des liens étroits avec Masaryk ${ }^{14}$, tout comme A. Meinong, qui est lui aussi privatdozent à la même université depuis 1878 . On sait également que Husserl était inscrit à une mineure en philosophie et qu'il a été examiné par R. Zimmermann et T. Vogt, quoique, durant ce séjour à Vienne, il n'a vraisemblablement pas fréquenté les leçons de Brentano ou celles de ses étudiants. Le 23 janvier 1883, il soutient avec succès sa thèse de doctorat «Contributions au calcul des variations».

Husserl retourne ensuite à Berlin en tant qu'assistant de Weierstraß dans l'intention d'y préparer sa thèse d'habilitation, projet qu'il abandonne rapidement en raison de la détérioration de l'état de santé de Weierstraß peu de temps après son arrivée à Berlin. Après une année de service militaire, il retourne à Vienne au semestre d'hiver 1884-1885 avec l'intention plus ou moins arrêtée d'entreprendre des études en philosophie. Masaryk, qui était en poste à Prague depuis 1882 en compagnie de Stumpf et de Marty, ainsi que Meinong, lequel avait obtenu un poste à Graz la même année, n'étaient donc plus dans l'environnement immédiat de Brentano. Dans ses «Souvenirs de Franz Brentano ", Husserl explique que c'est d'abord par curiosité qu'il assiste à une leçon de Brentano "pour entendre cet homme qui faisait tellement parler de lui dans la Vienne d'alors ${ }^{15}$.» L'impression que lui laissa cette première rencontre - tant du personnage que de sa manière de pratiquer la philosophie - lui confirma le bien-fondé des recommandations répétées de Masaryk et le convainquit de choisir la philosophie comme plan de vie:

12. C'est ce que confirme Malvine Husserl (p. 111) dans son mémoire sur Edmund Husserl: "Masaryk attira l'attention de manière enthousiaste sur Brentano, dont il était alors l'étudiant, et aurait souhaité volontiers que Husserl l'accompagne sur le champ à Vienne où il s'apprêtait à se rendre, afin d'y préparer son habilitation. "

13. C'est ce que Husserl explique clairement à Mahnke dans une lettre datée du mois de mai 1933, in Husserl, Briedwechsel, Bd III, p. 500.

14. C'est ce que confirme un passage de sa lettre de 1936 à Jancik, in Husserl Briefwechsel, Bd. VIII, p. 59.

15. Husserl, «Souvenirs de Franz Brentano», p. 153-154. 
Des cours de Brentano me vint pour la première fois la conviction que la philosophie aussi était un terrain de travail sérieux, qu'elle pouvait et même devait être traitée dans l'esprit de la science la plus rigoureuse, ce qui m'encouragea à la choisir comme métier pour la vie ${ }^{16}$.

Au cours des deux années passées à Vienne (1884-1886), Husserl assista à plusieurs séminaires de Brentano ${ }^{17}$ dont les préoccupations à cette époque concernaient principalement la psychologie descriptive, comme le note Husserl dans son mémoire de 1919:

Mais ce qui l'occupait surtout durant ces années, c'était d'une part des questions de psychologie descriptive, lesquelles firent le thème des leçons susmentionnées, et d'autre part des recherches sur la psychologie des sens, qui ont été publiées il y a quelques années seulement, et dont le contenu (du moins dans ses grandes lignes) m'est resté en mémoire sur la base de nos discussions à Vienne et à Saint-Gilgen ${ }^{18}$.

On sait que ces questions de psychologie descriptive ont été au cœur des recherches philosophiques de Husserl durant la période de Halle, et ce, jusque dans les Recherches logiques où la phénoménologie est définie comme une psychologie descriptive. Ce passage confirme en outre que Husserl a entretenu des liens étroits avec Brentano, tant sur le plan académique que sur le plan personnel, jusqu'à l'accompagner durant ses vacances d'été à Wolfgangsee. Ce mémoire nous apprend aussi que ces séminaires se poursuivaient parfois dans la maison de Brentano avec un cercle restreint de ses étudiants. Parmi les étudiants de Brentano qui ont transité de Vienne à Graz ou à Prague, et qui ont probablement appartenu à ce cercle, mentionnons Christian von Ehrenfels, Franz Hillebrand, Emil Arleth, Kazimierz Twardowski, Hans Schmidkunz et Alois Höfler ${ }^{19}$. Si l'on se fie à une remarque de von Ehrenfels ${ }^{20}$, Husserl n'a pas mis de temps à s'imposer durant ce court séjour, il est devenu la nouvelle star de Vienne.

Après ses études à Vienne auprès de Brentano, Husserl arrive à Halle à l'automne 1886 afin de compléter sa thèse d'habilitation sous la supervision de Stumpf, qui occupe un poste dans cette ville depuis 1884 . On sait que Brentano n'était pas habilité à diriger les thèses de ses propres étudiants, et c'est pourquoi ses premiers étudiants, Stumpf et Marty, ont dû s'exiler de Würzburg et poursuivre leurs études à Göttingen auprès de Hermann Lotze

16. Ibid., p. 154.

17. Cf. R. Rollinger: Husserl's Position in the School of Brentano, p. 17, pour la liste de ces cours.

18. Husserl, "Souvenirs de Franz Brentano », p. 157.

19. Cf. Fisette et Fréchette, «Le legs de Brentano», p. 53 sq.

20. Cf. la lettre d'Ehrenfels à Meinong datée du 22 février 1886, dans laquelle il parle "d'une nouvelle étoile dans le ciel philosophique appelée le Dr. Husserl», lettre citée in R. Fabian, «Leben und Wirken von Christian von Ehrenfels », p. 17. 
pour lequel Brentano avait beaucoup d'estime ${ }^{21}$. C'est ainsi que Brentano recommanda à Stumpf ce jeune étudiant enthousiaste dans l'espoir qu'il trouvât aussi en Georg Cantor, lequel était lui aussi en poste à Halle à ce moment, un interlocuteur privilégié pour les questions touchant l'aspect mathématique de ses travaux ${ }^{22}$. Ce qui ne veut pas dire que Stumpf n'agissait dans les circonstances que comme simple prête-nom. Au contraire, comme le rapporte Malvine Husserl dans son mémoire, durant le début de la période de Halle, "Stumpf fut le guide, le conseiller et l'ami paternel ${ }^{23}$.» Stumpf l'accueille donc avec enthousiasme et confirmera plus tard dans son autobiographie de 1924 que Husserl «est devenu un proche tant sur le plan scientifique que sur le plan personnel, d'abord comme étudiant, puis comme Dozent ${ }^{24}$ ». Cette date marque en effet le début d'une longue et fructueuse relation qui durera jusqu'à la mort de Stumpf en $1936^{25}$.

La première année d'étude à Halle est particulièrement intense pour le jeune philosophe: en plus de la préparation de sa thèse d'habilitation, il assiste aux leçons de Stumpf, et on lui impose plusieurs examens en vue de son habilitation à enseigner dans cette université. En effet, au semestre d'hiver de 1886-1887, il assiste aux leçons de Stumpf sur la psychologie et, au semestre d'été de 1887, il fréquente ses leçons sur la logique et l'encyclopédie de la philosophie ${ }^{26}$. Parce que Husserl possède un diplôme de doctorat de l'Autriche, l'université de Halle lui impose un examen de "nostrification » visant à reconnaître son diplôme autrichien, examen qui a lieu en juin 1887. Dans ce jury siègent le grand mathématicien Cantor et, ex officio, Stumpf qui l'examine sur plusieurs thèmes, dont la théorie des signes locaux de Lotze, l'histoire des théories de la perception de l'espace, et les relations entre la logique et les mathématiques ${ }^{27}$. Au début de juillet 1887, Husserl dispute sa thèse d'habilitation et présente sept thèses qu'il défend avec

21. Dans une lettre à Stumpf datée du 3 novembre 1867, Brentano lui explique pourquoi il a choisi de le recommander à Lotze: «Parce que je ne pourrais vous nommer aucun autre professeur de philosophie dont je ne tenais pas la doctrine pour fausse dans ses aspects essentiels, et parce que Lotze, malgré tout ce qui lui manque, est à maints égards remarquable. Notamment la méthode de sa philosophie, l'accent qu'il met sur l'expérience et l'observation, la manière par laquelle il utilise les résultats scientifiques, la prudence et la méticulosité avec lesquelles il avance ses thèses, le distinguent de manière avantageuse de la plupart des autres chercheurs de notre temps. Et je ne connais personne d'autre dont vous pourriez apprendre davantage à cet égard» (F. Brentano, Briefe an Carl Stumpf 1867-1917, p. 3).

22. Ibid., p. 83 sq.

23. K. Schuhmann, Husserl-Chronik, p. 114.

24. C. Stumpf, "Autobiographie», p. 266.

25. Cf. sa remarque sur Stumpf dans sa dernière lettre à Masaryk en date du 3 janvier 1935, Husserl, Briefwechsel, Bd. I, p. 119.

26. Sur cet aspect important de la formation de Husserl à son arrivée à Halle et les leçons de Stumpf, cf. K. Schuhmann, «Stumpfs Vorstellungsbegriff in seiner Hallenser Zeit ", Rollinger, op. cit., p. 83 sq., et D. Fisette «Stumpf and Husserl on Phenomenology and Descriptive Pychology».

27. C. Stumpf, in Gerlach (dir.), Husserl in Halle, p. 184. 
succès pour obtenir son diplôme de cette université. Son Habilitationsschrift, publié quelques mois plus tard, s'intitule "Sur le concept de nombre: analyses psychologiques", et son thème est l'origine psychologique des concepts fondamentaux de l'arithmétique. Dans son rapport, Stumpf note les qualités analytiques remarquables du candidat et souligne l'apport méthodologique important de la psychologie descriptive dans ce travail ${ }^{28}$.

\section{II}

Deux années après l'entrée en poste de Stumpf à Munich en 1889, Husserl publie le premier volume de Philosophie de l'arithmétique, qui est dédié à Brentano. Cet ouvrage s'appuie en bonne partie sur son Habilitationsschrift, et, comme l'indique A. Chrudzimski dans son article "Catégories formelles, nombres et conceptualisme. La première philosophie de l'arithmétique de Husserl », il est fortement empreint de la philosophie de Brentano. Un des principes brentaniens qui guident Husserl dans sa recherche sur l'origine du concept de nombre est l'empirisme des concepts suivant lequel tous nos concepts ont une origine intuitive et proviennent de l'expérience. La formation des concepts fondamentaux de l'arithmétique comme de tous les concepts en général est assurée, comme chez Brentano, par abstraction sur les opérations de dénombrement ou les actes psychiques correspondants, et ce sont donc des concepts d'ordre supérieur. On sait que Husserl a abandonné cette forme de conceptualisme dès après la publication de ce premier tome de l'ouvrage au profit d'une forme de platonisme inspiré de Bolzano et de Lotze, dont nous reparlerons plus tard. Mais comme le fait remarquer Chrudzimski, indépendamment des raisons qui ont pu motiver Husserl à se convertir au platonisme, cette première philosophie des mathématiques représente encore aujourd'hui une option viable du platonisme, en particulier pour ceux qui reconnaissent la valeur du programme philosophique de Brentano dans son ensemble. Dans tous les cas, on ne saurait discréditer la valeur de cet ouvrage au nom de l'objection du psychologisme logique et voir dans les travaux ultérieurs de Husserl durant cette période une remise en question du programme de Brentano.

Une des contributions originales de Philosophie de l'arithmétique porte sur ce que C. von Ehrenfels a appelé les "qualités de forme» dans un article portant le même nom ${ }^{29}$. Cet article a suscité une réaction immédiate

28. Ibid., p. 173. Quelques jours après la disputatio, Husserl présente une Probevorlesung sur le débat autour de la psychologie de l'introspection où les membres du jury étaient à nouveau Cantor et Stumpf, entre autres, et on rapporte que Husserl aurait adopté une attitude critique face aux thèses de Brentano sur cette question (cf. Schuhmann, op. cit., p. 20). Le 24 octobre 1887, Husserl prononce son discours inaugural "Sur les objectifs et la tâche de la métaphysique » et devient privatdozent à l'Université de Halle, un statut qu'il conservera jusqu'à son départ pour Göttingen en 1901.

29. C. von Ehrenfels "Sur les "qualités de forme" ", trad. fr. D. Fisette, in D. Fisette et G. Fréchette (dir.), À l'école de Brentano, Paris, Vrin, 2007, p. 227-262. 
de la part des philosophes et scientifiques et a donné lieu, dès sa parution en 1890, à un débat mémorable auquel ont pris part plusieurs étudiants de Brentano, dont Husserl ${ }^{30}$. Ehrenfels attribue l'origine de cette notion à la thèse de Mach dans Analyse des sensations, thèse suivant laquelle nous pouvons directement "sentir» (empfinden) des phénomènes complexes comme une mélodie ou une configuration spatiale aussi familière qu'un arbre, par exemple, qui "nous apparaît de prime abord comme un tout unitaire et indivisible». La question qui se pose à la psychologie descriptive est de savoir ce que sont ces configurations spatiales et sonores: "une simple synthèse d'éléments ou quelque chose de nouveau par rapport à ceux-ci, quelque chose qui se présente bien avec cette synthèse, mais qui en est pourtant distinct $^{31}$ ? ". L'objectif que se fixe Ehrenfels consiste à élucider et à définir la notion de qualité de forme et à "démontrer l'existence des objets correspondants dans la nature ${ }^{32}$.

Dans sa contribution à ce numéro, «Husserl et Stumpf sur la Gestalt et la fusion ", C. Ierna se penche sur la notion de moment figural que Husserl développe dans le chapitre XI de sa Philosophie de l'arithmétique afin d'expliquer les appréhensions indirectes de multiplicités. Son point de départ est une remarque de Husserl dans la troisième Recherche, où celui-ci revendique la priorité dans la découverte des objets d'ordre supérieur et des qualités de forme et soutient avec raison que les références de Husserl sont Stumpf et Mach. C'est ce que confirme en partie une note du chapitre XI de Philosophie de l'arithmétique dans laquelle Husserl mentionne l'article de von Ehrenfels dont il n'a reçu aucune influence puisqu'il ne l'a pas lu, mais il reconnaît sa dette à l'endroit de l'ouvrage de Mach, Analyse des sensations: "Puisque j'ai lu cet ouvrage du profond physicien aussitôt qu'il a paru, il est très possible que j’aie été moi aussi influencé dans la démarche de

30. Cf. M. G. Ash, Gestalt Psychology in German Culture 1890-1967, ainsi que D. Fisette et G. Fréchette, «Le legs de Brentano ", p. 13-160.

31. Ehrenfels, "Sur les "qualités de forme" ", p. 225 sq.

32. Sur réception de l'article d'Ehrenfels, Mach lui écrit qu'il a lui-même développé les idées que l'on retrouve dans cet article vingt ans auparavant, et on peut supposer, avec Smith, qu'il fait allusion ici à son article de 1865 intitulé «Bemerkungen zur Lehre vom räumlichen Sehen ». Dans ce texte, Mach se demande comment il est possible de reconnaître deux configurations spatiales (Gestalten) comme étant une seule et même figure, par exemple, comment on peut identifier une seule et même mélodie jouée dans deux clés différentes et par des instruments différents. Cette reconnaissance et cette similarité ne peuvent dépendre, soutient Mach, des qualités de représentation perceptuelles puisqu'elles sont différentes dans les deux cas. Nous pouvons interpréter cette remarque de Mach dans le sens d'un recours - nécessaire dans ce cas - à des sensations élémentaires additionnelles à l'extérieur de la sphère des représentations, à savoir à des sensations qu'il appelle des sensations musculaires ou kinesthèses: "Lorsque nous entendons la même mélodie dans deux clés différentes, notre appréhension de cette «identité » repose sur le fait que, même si la sensation des notes est perçue différemment, les mêmes sensations affectives sont impliquées dans les deux cas» (K. Mulligan \& B. Smith «Mach and Ehrenfels: The Foundations of Gestalt Theory», p. 126). 


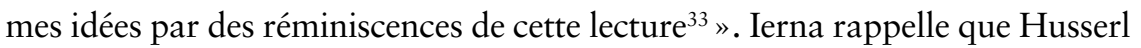
utilisait déjà la notion de Gestalt dans ses leçons de 1889-1890 sur le concept de nombre, comme le confirme le passage suivant dans lequel Husserl préfère la notion de moment de Gestalt à celle de moment figural qu'il utilise dans Philosophie de l'arithmétique:

Mais comment en arrive-t-on à une telle représentation symbolique? Réalisons-nous une disposition de points arbitraires au tableau ou bien imaginonsnous un nombre de points sur un cube, etc.? Qu'est-ce qui est donné en premier? Une certaine configuration de points. Il y a une intuition uniforme partant de laquelle nous pouvons remarquer ce moment de Gestalt et qui donne à tout le phénomène son aspect caractéristique. Cela constitue alors le cadre uniforme pour l'activité appréhensive: nous appréhendons un élément, allons ensuite vers un autre, puis encore vers un autre, etc. Or c'est le cadre extérieur, la Gestalt, l'uniformité de l'intuition qui nous permet de faire l'économie d'une collection véritable et qui rend possible la représentation symbolique d'une pluralité, laquelle est définie par cette intuition ${ }^{34}$.

Une fois établi que l'article d'Ehrenfels n'est pas aussi révolutionnaire qu'on l'a prétendu, Ierna examine le rôle de la notion de fusion dans la conception que se fait Husserl des moments figuraux, et il se demande si cette notion a les mêmes traits caractéristiques que ceux que Stumpf lui attribue dans sa psychologie.

\section{III}

La période de dix années qui sépare la publication du premier tome de $\mathrm{Phi}$ losophie de l'arithmétique (1891) des Recherches logiques (1900-1901) se subdivise en deux segments: le premier s'étend jusqu'au milieu des années 1890 et comprend les recherches de Husserl en vue de la rédaction du deuxième volume de Philosophie de l'arithmétique qui est annoncé dans la préface à cet ouvrage; le début du deuxième coïncide avec l'abandon de ce projet et se termine avec la publication de l'ouvrage le plus important de Husserl, à savoir les Recherches logiques. Quatre articles de ce numéro de Philosophiques portent sur différents aspects des travaux de Husserl durant le premier segment de cette période. Un de ces aspects concerne ses recherches dans le domaine de la géométrie et des représentations spatiales, ainsi

33. Husserl, Philosophie de l'arithmétique, p. 258. Signalons que la première édition de l'ouvrage de Mach est parue en 1886, date de l'arrivée de Husserl à Halle, et on sait que Stumpf a fait une recension positive de cet ouvrage la même année (C. Stumpf, "Rezension von "Ernst Mach, Beiträge zur Analyse der Empfindungen" ", Deutsche Literaturzeitung, vol. 27, 1886, p. 947-948). Mais dans les ouvrages ultérieurs de Husserl où il est question de ces phénomènes, ce n'est plus le nom de Mach qui est mentionné, mais bien ceux d'Ehrenfels et de Meinong, et Husserl utilisera de préférence la notion de moment d'unité à partir de 1894 dans ses «Études psychologiques".

34. Husserl, «Vorlesung Über den Begriff der Zahl (WS 1889/90)», p. 298. 
que le projet de la rédaction d'un Raumbuch ${ }^{35}$. L'article de C. Hill porte sur la genèse de la théorie des multiplicités (Manningfaltigkeiten) définies que Husserl élabore durant cette période. Elle soutient que cette théorie a permis à Husserl de surmonter de nombreux problèmes qui grevaient le programme "psychologique » de son ouvrage de 1891, dont le problème de l'imaginaire qui, comme l'indiquait Husserl dans la préface de Philosophie de l'arithmétique, représentait une des tâches principales assignées aux recherches en vue du deuxième volume de l'ouvrage. L'importance qui revient à ce problème durant cette période pré-phénoménologique est confirmée par plusieurs remarques de Husser ${ }^{36}$, et notamment dans ses Prolégomènes où il est formulé de la manière suivante: "Comment, par exemple, dans le domaine des nombres, des concepts impossibles (irréels [wesenlose]) peuvent-ils être traités méthodiquement comme des nombres réels ${ }^{37}$ ?». Dans une conférence importante intitulée "L'imaginaire en mathématiques ", prononcée en 1901 devant la Société mathématique de Göttingen à laquelle appartenaient entre autres les célèbres mathématiciens D. Hilbert et F. Klein, Husserl formule le problème de l'imaginaire de la manière suivante:

Soit un domaine donné d'objets, dans lequel, par la nature particulière des objets, sont déterminées des formes de jonction et de relation qui s'énoncent dans un certain système d'axiomes A. Sur le fondement de ce système, donc sur le fondement de la nature particulière des objets, certaines formes de jonction n'ont pas de signification réelle, c'est-à-dire que ce sont des formes de jonction qui sont absurdes [widersinnige]. De quel droit ce qui est absurde peut-il être utilisé dans le calcul, de quel droit ce qui est absurde peut-il être employé par la pensée déductive comme si c'était quelque chose de concordant? Comment est-il possible d'expliquer qu'on puisse opérer avec ce qui est absurde selon des règles, et que, si ce qui est absurde se situe en dehors des propositions, les propositions obtenues soient justes ${ }^{38}$ ?

La solution qu'il propose à ce problème réside dans sa théorie des multiplicités qu'il conçoit comme un domaine ou région d'objets «délimité en tant que sphère quelconque d'objets en général, peu importe qu'ils soient réels ou idéaux, pour lesquels sont valables des propositions fondamentales de telles et telles formes. Nous appelons un domaine d'objets ainsi défini une multiplicité déterminée, mais définie formellement ${ }^{39}$.» Le concept de définitude

35. Cf. Husserl, Hua XXI.

36. Cf. notamment dans le premier livre des Idées directrices (Hua III, p. 153), Logique formelle et logique transcendantale (Hua XVII, p. 101) et les Prolégomènes (Hua XVIII, 251).

37. Husserl, Hua XIX/1, p. 251.

38. Husserl, «Husserls Manuskripte zu seinem Göttinger Doppelvortrag von 1901 », p. 93.

39. Ibid., p. 495. 
(Definitheit) est à comprendre dans le sens de l'axiome de saturation de Hilbert $^{40}$ :

Le système d'axiomes définissant formellement une telle multiplicité se distingue par le fait que toute proposition (toute forme de proposition) devant être construite conformément à la grammaire pure logique, à l'aide des concepts (des formes de concepts naturellement) qui interviennent dans ce système axiomatique, est ou "vraie » (c'est-à-dire une conséquence analytique — purement déductive des axiomes) ou «fausse " (c'est-à-dire est une contradiction analytique): tertium non datur ${ }^{41}$.

Il s'ensuit que toutes les propositions, y compris les propositions contenant des nombres imaginaires, sont soit des conséquences des axiomes, soit en contradiction avec eux. D'où la possibilité d'opérer librement avec des concepts imaginaires et la solution à notre problème: "Quand les systèmes sont "définis", alors le calcul avec des nombres imaginaires ne peut jamais conduire à des contradictions ${ }^{42}$.» Telle est la position définitive de Husserl sur cette question et, comme le remarque Hill, cette théorie représente le cœur de la doctrine de la science et de la logique pure dont nous reparlerons ci-dessous ${ }^{43}$.

Tout aussi importantes à cette époque sont les recherches de Husserl dans le domaine de la psychologie descriptive, comme le montrent les études originales de R. Rollinger, G. Fréchette et R. Brisart qui sont reproduites dans ce numéro. La théorie des assomptions, que Husserl élabore dans un texte publié à titre posthume sous le titre de «Intentionale Gegenstände » (Objets intentionnels), est ce qu'il y a de commun à ces trois études ${ }^{44}$. Dans son texte "Quelques aspects de la première théorie du jugement de Husserl ", Rollinger examine la théorie du jugement élaborée par Husserl dans deux manuscrits rédigés en 1893-1894 et publiés récemment sous le titre "Versuch über den Ursprung der Begriffe "Notwendigkeit" [und] "notwendige Folge", über hypothetisches und kausales Urteil »"45 (1893) et "Eigentliche

40. Pour le rapprochement de ce concept de «définitude» avec l'axiome de saturation de Hilbert, cf. Husserl, Hua XVII, $\mathbb{} 31$ et Hua III/1, p. 153.

41. Husserl, Hua XVII, p. 100.

42. Husserl, Hua XVII, p. 101.

43. La logique et la philosophie des mathématiques de Husserl ont fait l'objet de nombreux ouvrages récents dont celui de S. Centrone, Logic and Philosophy of Mathematics in the Early Husserl; cf. aussi R. Tieszen, Phenomenology, Logic, and the Philosophy of Mathematics, Atten, Mark van, Brouwer meets Husserl. On the Phenomenology of Choice Sequences et V. Peckhaus (dir.) Oskar Becker und die Philosophie der Mathematik.

44. Une partie de ce texte a été publiée par B. Rang dans le volume XXII des Husserliana, mais la version de ce texte que Husserl prévoyait publier, soit la version complète, a été éditée par Karl Schuhmann sous le titre «Husserls Abhandlung "Intentionale Gegenstände"; Edition der ursprünglichen Druckfassung ".

45. Husserl, Hua XL, p. 1-30. 
und uneigentliche Urteile ${ }^{46}$ (1893-1894). Il remarque que cette première théorie du jugement de Husserl se démarque sur plusieurs points de celle de Brentano et de Stumpf, et d'abord sur la classification des actes en représentation, jugement et sentiment. Car Husserl reconnaît une classe additionnelle d'actes entre les représentations et les jugements qu'il appelle justement des «assomptions ». Comme l'explique Husserl dans le premier de ces deux manuscrits de recherche:

La position hypothétique, l'acte d' «assumer", est un comportement psychique particulier face à l'état de choses placé («représenté») objectivement et relève de la même irréductibilité psychologique que l'acte de reconnaître, de rejeter, de présumer, de douter, etc. De la réflexion sur ce comportement psychique résulte la paire corrélative des concepts de l'acte d'assumer et de ce qui est assumé, et de ce dernier concept, par abstraction toutefois de l'individuel et du subjectif, résulte le concept d'objet d'assomption, d'hypothèse ${ }^{47}$.

Ce passage, comme plusieurs autres, montre que Husserl en est venu à l'idée même d'assomption bien avant que Meinong l'élabore systématiquement au début des années 1900, notamment dans son ouvrage classique Über Annabmen (Sur les assomptions) ${ }^{48}$.

Le texte "Objets intentionnels» de 1894 nous informe sur le genre de problèmes que Husserl cherchait à solutionner avec sa théorie des assomptions. Rappelons d'abord que ce texte est une réaction à la publication, en 1894, de l'opuscule de K. Twardowski Sur la théorie du contenu et de l'objet des représentations. Une recherche psychologique ${ }^{49}$, dont le thème central est le problème des représentations sans objet ${ }^{50}$, problème qui n'est pas étranger à celui de l'imaginaire en mathématiques. Ce même problème est abordé dans la deuxième étude de son article, publié lui aussi en 1894, qui a pour titre "Études psychologiques pour la logique élémentaire", dans lequel il introduit sa théorie des touts et des parties et la double distinction

46. Husserl, Hua XL, p. 31-51 et les appendices à ce texte, p. 51-67.

47. Husserl, Hua XL, p. 18 sq.

48. Sur les assomptions chez Husserl et Meinong, cf. Rollinger, Husserl's Position in the School of Brentano p. 186-199.

49. K. Twardowski, Zur Lehre vom Inhalt und Gegenstand der Vorstellungen: eine psychologische Untersuchung.

50. Une lettre de Husserl à Meinong (Briefwechsel, Bd. I, p. 139 sq.) indique clairement que ce texte porte principalement sur la position que défend Twardowski dans son ouvrage. Husserl a annoté ce manuscrit à deux reprises, soit en 1902 puis en 1906 à l'occasion d'une relecture de l'ouvrage de Meinong Über Annabmen, et il semble que Husserl l'ait offert à Meinong (voir la lettre de Meinong du 10-04-1902, Husserl, Briefwechsel, Bd. I, p. 145 sq.) et à Daubert (lettre à Daubert du 17-11-1904, Husserl, Briefwechsel, Bd. II, p. 41 sq.). Pour ce qui est de la recension de 1896, Husserl l'aurait transmise à Twardowski, mais seulement en 1928, comme en témoigne une lettre de Twardowski à Husserl datée du 17-08-1928 (Husserl, Briefwechsel, Bd. I, p. 182 sq.). 
entre, d'une part, intention et remplissement ${ }^{51}$, et d'autre part, représentation représentative (Repräsentation) (en tant que "bloss intendieren ${ }^{52}$ ) et intuition (Anschauung) ( «als immanente Inhalte wirklich in sich fassen ${ }^{53}$ »). Les représentations comme celles d'un carré rond et d'un fer de bois qui renferment des incompatibilités sont des exemples de re-présentations qui sont dirigées sur quelque chose d'impossible. D'où la distinction entre trois classes de représentations, à savoir celles qui peuvent être factuellement remplies, celles qui peuvent être idéalement remplies, et celles qui sont impossibles à remplir. Ce sont précisément ces dernières qui appartiennent à ce que Bolzano ${ }^{54}$ appelle la classe des représentations "sans objet » ou représentations impossibles, lesquelles constituent le thème central de «Objets intentionnels ». C'est en réponse à ce problème que Husserl élabore une première version de sa propre théorie de l'intentionnalité que l'on retrouve dans la cinquième Recherche, de même que sa théorie des assomptions.

Un des aspects importants du problème des objets intentionnels concerne la théorie immanentiste de l'intentionnalité de Brentano dans sa Psychologie d'un point de vue empirique de 1874. Un des apports de Twardowski à ce qu'il est maintenant convenu d'appeler le problème de l'intentionnalité réside dans la fameuse distinction entre le contenu et l'objet d'un acte psychique, bien qu'il conserve la thèse initiale de l'inexistence intentionnelle par laquelle Brentano caractérise les phénomènes mentaux. Mais Twardowski, tout comme Husserl et la plupart des étudiants de Brentano, présuppose une certaine compréhension de la thèse de Brentano qui semble correspondre à ce que $\mathrm{M}$. Antonelli appelle, dans l'article que nous reproduisons ici, l'interprétation ontologique de l'intentionnalité chez Brentano. Un des défenseurs les plus connus de cette interprétation dans les études brentaniennes est R. Chisholm, qui soutient que la définition initiale de l'intentionnalité dans la Psychologie de 1874 est grevée d'une équivocité entre les deux thèses suivantes: la thèse psychologique suivant laquelle la référence à un objet ou ce qu'on appelle la directionnalité des phénomènes psychiques; et la thèse ontologique qui veut que les objets des actes psychiques possèdent un statut ontologique particulier, à savoir l'inexistence intentionnelle. D'où l'idée que l'intentionnalité chez Brentano ne désigne pas une relation spéciale des phénomènes psychiques à des objets ordinaires, mais bien une relation ordinaire à des objets dont le trait caractéristique principal est d'exister (ou de subsister) dans les actes. On connaît les arguments formulés par les étudiants de Brentano contre la thèse ontologique: cette théorie de l'intentionnalité serait en principe incapable de rendre compte de

51. Husserl, "Psychologische Studien zur elementaren Logik», Husserl, Hua VI, p. $92-123$.

52. Husserl, Hua XXII, p. 107.

53. Husserl, Hua XXII, p. 107.

54. Bolzano, Wissenschaftslehre, $\mathbb{} 70$. 
la différence entre les pensées concernant les objets intentionnels et celles concernant les objets réels. Mais comme le montre Antonelli dans son texte, Brentano se défend d'avoir jamais défendu cette thèse ontologique. Par exemple, dans une lettre à Marty du 17 mars 1905, il écrit à ce propos:

Je n'ai jamais soutenu que l'objet immanent puisse être identifié avec l'objet représenté. La représentation a la «chose» pour objet et non pas la «chose représentée». La représentation d'un cheval, par exemple, n’a pas comme objet immanent un "cheval représenté » mais bel bien un "cheval» (le seul qu'on puisse véritablement appeler ainsi) ${ }^{55}$.

Doit-on accorder crédit à Brentano de cette interprétation rétrospective de sa propre théorie de l'intentionnalité, et par voie de conséquence admettre que ses étudiants Stumpf, Meinong, Husserl et Twardowski n'auraient pas compris le sens même de la doctrine du philosophe de $V_{i e n n e}{ }^{56}$ ? S'appuyant sur une analyse précise du corpus brentanien pertinent sur cette question, Antonelli soutient que Brentano n'a jamais adhéré à la thèse ontologique qu'on lui attribue et s'oppose lui aussi à l'idée d'un prétendu tournant dans la pensée de Brentano sur cette question ${ }^{57}$.

Deux études du présent numéro examinent plus spécifiquement le thème des assomptions dans le texte de Husserl "Objets intentionnels": R. Brisart «La théorie des assomptions chez le jeune Husserl» et G. Fréchette "Géométrie, fiction et discours sous hypothèse: Husserl et les objets intentionnels en 1894 », dans lequel il expose habilement et précisément les tensions dans ce texte remarquable de Husserl entre les doctrines (des actes) de Brentano et celle (de la signification) de Bolzano ${ }^{58}$. Une des thèses importantes de Husserl qui sont discutées dans ces articles concerne l'identité du réel et de l'imaginaire dans le domaine de la psychologie et des mathématiques. Le discours sur la représentation des objets impossibles est relégué au rang de manière elliptique et impropre de parler des objets mathématiques, par exemple, et la solution que propose Husserl à ce problème repose sur l'idée que tous les énoncés sur les objets mathématiques et tous les énoncés mathématiques en général se tiennent sous une assomption générale qui n'est jamais explicite et suivant laquelle les axiomes ou propositions de base ont un caractère hypothétique. Comme l'explique Husserl dans «Objets intentionnels », dans le cas du concept d'espace en géométrie le mathématicien est indifférent à l'existence ou à la non-existence de l'espace, comme il l'est à l'égard de l'existence d'une figure quelconque. Comme dans la mythologie,

55. Passage cité dans l'article d'Antonelli reproduit dans ce numéro.

56. Cf. notamment Fisette et Fréchette, «Le legs de Brentano».

57. Voir l'ouvrage d'Antonelli Seiendes, Bewusstsein, Intentionalität im Frühwerk von Franz Brentano et la littérature très abondante sur cette question dont discute Antonelli dans cet ouvrage.

58. Voir l'ouvrage récent de Fréchette Gegenstandslose Vorstellungen. Bolzano und seine Kritiker. 
les propositions d'existence sur l'espace ou sur la suite des nombres, le mathématicien les assume, c'est-à-dire que ces propositions, comme l'ensemble des propositions mathématiques, se tiennent sous une assomption générale que l'on peut expliciter de la manière suivante: "À supposer qu'il y ait un espace, une multiplicité de tel et tel genre déterminé (défini exactement dans les soubassements), il existe alors en elle telles et telles formations, pour celles-ci sont valables telles et telles propositions, et ainsi de suite $^{59}$.» Il en va de même dans le domaine de l'arithmétique formelle dont les soubassements sont des hypothèses qui servent à la construction d'un domaine mathématique fermé comme l'algèbre; ces propositions fondatrices forment «le système infiniment ramifié des conséquences formelles de ces soubassements, qui peuvent en dériver dans une déduction ${ }^{60}$ ». Fréchette et Brisart se demandent ce qu'il advient de la théorie des assomptions après 1894, et ce dernier en fait la genèse jusque dans les derniers travaux de Husserl.

\section{V}

Durant le dernier segment de la période de Halle, qui marque l'abandon définitif du projet initial annoncé dans sa Philosophie de l'arithmétique, Husserl entreprend au milieu des années 1890 de nouvelles recherches qui mèneront comme on le sait à la publication des Recherches logiques (19001901). Ce changement dans l'orientation des recherches de Husserl durant cette période a suscité beaucoup d'intérêt dans les études husserliennes, et on a cherché une explication, sinon une cause, dans la critique du psychologisme que Frege lui a adressé dans son compte rendu de 1894 de Philosophie de l'arithmétique ${ }^{61}$. Plusieurs indications attestent en effet que ce changement important s'est produit au milieu des années 1890, et il concerne non seulement les relations entre psychologie et logique (et mathématiques), mais avant tout la conception qu'il se fait de la logique. D'autres informations nous permettent de situer plus précisément ce tournant entre 1894 et 1896, c'est-à-dire entre l'abandon définitif du projet de Philosophie de l'arithmétique et l'élaboration des éléments essentiels des Prolégomènes à la logique pure dans les leçons de logique de 1896, dont nous reparlerons. On ne peut pas exclure d'entrée de jeu que la critique pertinente que lui adresse Frege dans son compte rendu n'ait pas suscité quelques inquiétudes chez Husserl, d'autant qu'elles vont dans le sens de celles que lui-même adresse à Twardowski dans "Objets intentionnels» et dans un compte rendu de son ouvrage de 1894. Cependant, le sens même de la critique que Husserl adresse au psychologisme logique dans les Prolégomènes présuppose la logique pure et la doctrine de la science qu'il élabore au milieu des années 1890, et ses

59. Husserl, Hua XXII, 328.

60. Ibid., p. 324.

61. G. Frege, «Rezension von: E. Husserl, Philosophie der Arithmetik I». 
références à cet égard sont principalement Bernard Bolzano et Hermann Lotze $^{62}$.

Dans sa contribution substantielle à ce numéro de Philosophiques «Bolzano et (le jeune) Husserl sur l'intentionnalité », Wolfgang Künne examine la réception de l'œuvre maîtresse de Bolzano, Wissenschaftslehre, dans l'école de Brentano, et plus particulièrement chez Husserl durant la période de Halle. Après avoir exposé de manière élégante la doctrine bolzanienne des représentations et des propositions en soi de Bolzano, Künne montre que cette doctrine est paradigmatique de la conception que se fait Husserl non seulement de la signification comme species, mais encore des contenus intentionnels dans ses Recherches logiques. Le fil conducteur de son article est un passage de la correspondance de Husserl avec Brentano dans laquelle il explique à son maître inquiet les tenants et aboutissant de sa critique du psychologisme et l'importance qu'il attribue à Bolzano et à Lotze dans son tournant anti-psychologiste. Dans une lettre du 27 mars 1905, Husserl écrit à Brentano:

Du reste, mes Prolégomènes ne sont pas dirigés contre vous et vos étudiants. Au fond, je crois qu'il n'existe pas de grande différence entre nous sur ces questions plus générales, il m'importe seulement de mettre l'accent sur des distinctions spécifiques que vous croyez pouvoir éviter. Entièrement éloignée de moi, dois-je ajouter, est l'exploitation mystico-métaphysique des «Idées ", des possibilités idéales, etc. De même les représentations et propositions en soi de Bolzano ne sont pas actuelles [realisiert]. Ces conceptions de Bolzano ont exercé une grande influence sur moi, tout comme l'interprétation par Lotze de la doctrine platonicienne des Idées. Cela dit, je ne peux pourtant pas le considérer comme le "maître » et "guide » au regard de ce que j’ai présenté dans mes Recherches logiques. Ce que je propose, ce sont des fragments d'une théorie de la connaissance et d'une phénoménologie de la connaissance, et ces deux dernières sont étrangères à Bolzano. Il fut un éminent mathématicien et un esprit logique, mais les analyses conceptuelles les plus fines et les théories logicoformelles côtoient chez lui une théorie de la connaissance tout simplement

62. Dans son ouvrage récent sur la philosophie de Husserl, Mohanty écrit à ce propos: «Bolzano (and not Twardowski) and Lotze (not Frege) remain the major influence which led Husserl to his theory of logic in the Logical Investigations» (Mohanty, 2008, p. 50). En ce qui concerne la question du psychologisme, rappelons que Stumpf, l'étudiant de Lotze, a publié en 1891 le premier traité sur cette question ( «Psychologie und Erkenntnistheorie»), et c'est à cet article que se réfère Husserl quand il critique le psychologisme logique dans ses Prolégomènes. Le sens que prend la critique du psychologisme dans ce traité de Stumpf est pertinent dans le présent contexte dans la mesure où, d'une part, elle montre que dans Philosophie de l'arithmétique la psychologie descriptive ne semble pas en cause dans cette critique, d'autre part, elle explique en partie l'apparente tension entre la critique du psychologisme dans le premier ouvrage et le rôle central de la psychologie descriptive dans les six recherches qui composent le deuxième tome des Recherches logiques. 
naïve. On ne retrouve aucune trace chez lui (comme chez Lotze) de l'idée d'une élucidation purement phénoménologique de la connaissance ${ }^{63}$.

Cette double influence de Bolzano et de Lotze est bien documentée dans l'œuvre de Husserl, et notamment dans son compte rendu d'un ouvrage de M. Palagyi de 1903, dans lequel on retrouve plusieurs passages qui confirment les propos de cette lettre à Brentano, et d'abord celui-ci:

C'est d'abord l'assimilation intime de ces réflexions de Lotze [autour de son interprétation de la théorie platonicienne des idées], incomplètement clarifiées à mon avis, qui m'a donné la clé pour pénétrer dans les conceptions de Bolzano, étranges et d'abord incompréhensibles dans leur naïveté phénoménologique, et dans les trésors de sa Théorie de la science ${ }^{64}$.

Les conceptions de Bolzano auxquelles il fait allusion dans ce passage sont ses propositions en soi qu'il conçoit dans ce même compte rendu comme des contenus de jugements ou sens d'énoncés:

[P]ar "proposition en soi", il ne faut entendre rien d'autre que ce que l'on désigne comme le «sens» de l'énoncé dans le langage quotidien, idéalement objectivant, et que l'on déclare être une seule et même chose, quand on dit par exemple de différentes personnes qu'elles affirment la même chose; ou bien aussi ce que l'on appelle en science purement et simplement une proposition, ainsi la proposition qui porte sur la somme des angles, personne n'ayant ici l'idée de penser aux vécus de jugement de qui que ce soit ${ }^{65}$.

Ce passage explique bien le caractère objectif de ces propositions et leur indépendance par rapport aux vécus subjectifs, mais il ne rend pas compte du caractère idéal de la signification en général et de la conception particulière que s'en fait Husserl dans les Recherches logiques comme essences d'actes. C'est ici qu'intervient Lotze et son interprétation des Idées platoniciennes en termes de Geltung et d'idéalité:

La proposition, écrit Husserl, se rapporte donc à chacun des actes du jugement auxquels elle appartient en tant qu'elle est leur visée identique, de la même façon par exemple que l'espèce du rouge appartient aux cas particuliers du «même» rouge. Si l'on se fonde sur cette conception, alors la théorie de Bolzano selon laquelle les propositions sont des objets mais n'ont cependant pas d' « existence ", acquiert la signification suivante, qu'il est facile de comprendre: c'est qu'elles ont l'être «idéal» ou la valeur d' "objets généraux» (donc le même être que celui qui est établi par exemple dans les "preuves d'existence » en mathématiques), mais pas l'être réel des choses ou des moments dépendants des choses, des particularités temporelles en général ${ }^{66}$.

63. Husserl, Briefwechsel, Bd. I, p. 39.

64. Husserl, Hua XXII, p. 152-161; trad. fr. p. 216.

65. Ibid.

66. Ibid., p. 217. 
Ces passages confirment, d'une part, l'importance de la Geltung de Lotze dans son interprétation des propositions et des lois de la logique comme des objets idéaux, et, d'autre part, le rôle de Lotze dans ce tournant antipsychologiste. Car l'argument central des Prolégomènes contre le psychologisme logique s'appuie entièrement sur l'idéalité des lois et principes de la logique et leur irréductibilité aux lois empiriques de la psychologie. Cette conception de la signification et des propositions en soi de Bolzano en termes d'idéalité est tirée directement de l'interprétation des Idées platoniciennes dans le troisième livre de la grande logique de Lotze, dans le chapitre intitulé "Le monde des idées ${ }^{67}$. Notons enfin que l'importance qui revient à la logique de Lotze ne se réduit pas à son interprétation des Idées platoniciennes, et corrélativement à son interprétation des propositions en soi de Bolzano. En effet, dans ses recherches du milieu des années 1890, qui aboutiront à l'idée de logique pure et au projet d'une doctrine de la science notamment dans ses leçons de 1896 sur la logique —, Husserl reconnaît sa dette à l'endroit de la Wissenschaftslehre de Bolzano, mais il souligne aussi l'importance de l'idée de Lotze dans sa logique de 1874 suivant laquelle l'arithmétique n'est qu'un morceau (ein Stück) de la logique. Un passage de ces leçons confirme la grande importance qui revient à cette idée pour ses propres recherches:

67. L'étude la plus détaillée de Husserl sur la logique de Lotze est un manuscrit daté 1895-1897 auquel il se réfère d'ailleurs dans ses Prolégomènes et qu'il entendait publier en annexe à ses Recherches logiques. La datation de ce manuscrit corrobore la thèse du tournant antipsychologiste au milieu des années 1890, tournant qui coïncide entre autres choses avec son adhésion au platonisme au sens de Lotze. Dans ce manuscrit, comme dans ses annotations en marge de son exemplaire de la logique de Lotze, la plupart des remarques de Husserl portent sur le troisième livre de la grande logique intitulé «Vom Erkennen» qui porte sur la question de savoir dans quelle mesure nos pensées peuvent prétendre à une connaissance de ce que nous présupposons être les corrélats objectifs et les causes de nos représentations. Husserl s'intéresse plus particulièrement dans ce ms à la distinction tranchée que fait Lotze dans la $\mathbb{} 314$ entre le subjectif et l'objectif ou, comme l'écrit celui-ci, entre "un contenu signifiant en soi quelque chose que nous nous représentons, d'une simple affection que nous subissons ". Il commente longuement certains passages de la $\$ 316$ sur le sens de la notion de Geltung. Dans tout le reste du manuscrit, Husserl essaye de montrer que, dans les chapitres suivants de sa logique, Lotze n'est pas toujours cohérent avec son platonisme et sa conception objectiviste des propositions et de la signification en général; il cite plusieurs passages dans lesquels il lui reproche de véhiculer certains préjugés subjectivistes hérités de Herbart et de Kant. Ce sont ces mêmes préjugés qu'il dénonce dans les Prolégomènes, notamment dans la théorie de la connaissance de Lotze qu'il qualifie d'hermaphrodite ou encore d'hybride contradictoire de logique pure et de logique psychologiste, «un produit de la demi-mesure, répugnant aux conséquences ultimes» (p. 70). Autrement dit, suivant le diagnostic de Husserl, Lotze n'aurait pas vu toute la portée philosophique de son interprétation de la théorie platonicienne des idées et n'aurait pas réussi à en tirer toutes les conséquences en ce qui concerne le psychologisme et la théorie de la connaissance. Au contraire, comme l'explique Husserl dans «Esquisse d'une préface », après avoir établi dans toute sa pureté sa théorie des Idées, Lotze rechute dans une forme d'anthropologisme où les pensées dépendent des vécus du sujet connaissant. D'où les nombreux reproches que lui adresse Husserl un peu partout dans son œuvre. Sur cette question, cf. D. Fisette: "Husserl et Lotze». 
Nous allons donc devoir nous familiariser avec la conception d'abord étrange de Lotze selon laquelle l'arithmétique n'est purement et simplement qu'un fragment relativement indépendant et depuis longtemps hautement développé de la logique. En effet, elle représente aussi dans une perspective pratique l'instrument le plus grandiose que l'esprit humain ait produit aux fins de la déduction ${ }^{68}$.

La dernière partie de l'article de Künne porte sur ce qu'il appelle le principe de Bolzano suivant lequel les questions sont des espèces de proposition dont la vérité se prête aussi à une évaluation, et il examine la critique que Husserl adresse à ce principe dans le dernier chapitre de ses Recherches $\operatorname{logiques}^{69}$.

\section{VI}

Le premier volume des Recherches logiques a été publié en 1900. On rapporte que Husserl était réticent à l'idée de publier cette version de ses recherches et que le manuscrit n'est parvenu à la maison d'édition que parce que Stumpf lui-même l'avait remis à celle-ci ${ }^{70}$. Husserl a dédié l'ouvrage à Stumpf au regard de sa vénération et de son amitié ${ }^{71}$. On mesure l'originalité et la portée philosophique de cet ouvrage monumental de plus de mille pages à l'influence majeure qu'il a exercée sur la philosophie germanophone jusqu'au début des années 1930. Nous avons déjà mentionné sa contribution dans les Prolégomènes à la logique pure en ce qui a trait à sa critique du psychologisme logique et à sa théorie de la science. Mais la contribution de cet ouvrage ne s'arrête pas là. En effet, dans les six recherches publiées dans les deux autres volumes de l'ouvrage, Husserl livre une partie des résultats de sa recherche durant la période de Halle sur des questions aussi diversifiées que le langage et la signification (Recherche I), l'abstraction et la formation des concepts (Recherche II), la méréologie (Recherche III), la grammaire (Recherche IV), l'intentionnalité des états mentaux (Recherche V), sa théorie de la connaissance (Recherche VI) et la perception interne de Brentano (Appendice). Parmi les nombreuses questions qui ont fait l'objet de discussions depuis la parution de cet ouvrage il y maintenant plus de cent ans, celle qui porte sur l'unité de l'ensemble des éléments de cet œuvre présente un intérêt particulier à la lumière de la disputatio autour de Husserl, l'ouvrage récent de D. W. Smith. Il existe en effet une tension entre les arguments anti-

68. Husserl, Logik. Vorlesung 1896, p. 271-272.

69. Cf. W. Künne «Husserl and Bolzano on Questions».

70. Cf. K. Schuhmann, op. cit., p. 57-58.

71. La dette de Husserl à l'égard de Stumpf dans cet ouvrage et durant la période de Halle en général est considérable, et elle touche des thèmes aussi importants que le psychologisme, la perception sensible, l'abstraction et la formation des concepts, la théorie des touts et des parties, les contenus de jugements et les Sachverhalten, les relations primaires et la notion de fusion, etc. Cf. D. Fisette "Stumpf and Husserl on Phenomenology and Descriptive Psycho$\log { }^{\prime}$. 
psychologistes des Prolégomènes et la phénoménologie pratiquée dans les deux autres volumes de l'ouvrage que Husserl définit comme une psychologie descriptive dans un sens très proche de celui que lui prêtait Brentano. Mais le problème de l'unité qui intéresse Smith soulève la question plus générale de savoir si l'ensemble des parties qui forment le corps des Recherches logiques est un tout homogène ou bien la somme de recherches isolées. Autrement dit, qu'est-ce qui assure l'unité des différents aspects de ces Recherches tels la logique, l'ontologie, la théorie de la connaissance et la phénoménologie, par exemple? Smith défend la thèse suivant laquelle, par-delà les Recherches logiques, l'ensemble de la philosophie de Husserl est tout aussi systématique que celles d'Aristote ou de Kant, et que c'est sa théorie de l'intentionnalité qui soude ensemble les pièces de ce "holisme structuré »"2 . Cette thèse de Smith est discutée par J. J. Drummond, E. Marbach et J.-M. Roy qui ont pris part à la disputatio, dont nous reparlerons ci-dessous.

Trois articles que nous publions dans ce numéro abordent des thèmes qui sont directement liés aux Recherches logiques: les articles de J.-F. Courtine, "Réduction, construction, destruction», et de D. Seron, "La critique de la psychologie de Natorp dans la Ve Recherche logique de Husserl ", se penchent principalement sur les relations entre Husserl et le néokantien P. Natorp, tandis que dans son article "L'intuition est-elle un concept univoque?»D. Pradelle aborde la question de l'unité des concepts d'intuition, d'évidence et de remplissement dans les Recherches logiques et au-delà. Les études de Seron et de Courtine soulèvent toute la question du rapport de Husserl aux différents courants néokantiens au XIX ${ }^{e}$ siècle dont les deux principaux sont premièrement le néokantisme de Bade, représenté principalement par W. Windelband, l'étudiant de Lotze, ainsi que H. Rickert, B. Bauch, lequel a supervisé la thèse de R. Carnap, et E. Lask, qui a suscité beaucoup d'intérêt de la part de l'auteur de Sein und Zeit ${ }^{73}$; le deuxième courant est le néokantisme de Marbourg, auquel appartient notamment Natorp. Comme le remarque Seron, les échanges qui ont marqué les relations Husserl-Natorp touchent plusieurs questions centrales de la phénoménologie, dont les plus importantes pour la période de Halle sont les suivantes: les problèmes de l'espace et du temps qui sont traités dans la correspondance entre 1897-190174; le reproche de psychologisme adressé au néokantisme dans les Prolégomènes, dont Natorp a fait la recension en $1901^{75}$; la critique que Natorp adresse à la notion d'ego dans la cinquième Recherche, et l'influence qu'il aurait exercé sur le tournant transcendantal de la phéno-

72. Sur la question de l'unité des Recherches logiques, cf. l'article de D. W. Smith «The Unity of the Logical Investigations: Then and Now".

73. Sur l'école néokantienne de Bade dans son rapport à Lotze et à l'école de Brentano, cf. D. Fisette "La philosophie de Carl Stumpf, ses origines et sa postérité », p. 51 sq.

74. Cf. Husserl, Briefwechsel, Bd. V., p. 39 sq.

75. P. Natorp, "Zur Frage der logischen Methode. Mit Beziehung auf Edmund Husserls Prolegomena zur reinen Logik». 
ménologie; enfin, le thème psychologique de la perception interne et la critique que Husserl adresse à l'ouvrage de Natorp, Introduction à la psychologie ${ }^{76}$, dans les premières $\mathbb{S} \mathbb{S}$ de la cinquième Recherche. L'article de Seron se concentre sur cette dernière question tandis que Courtine insiste davantage sur la critique que Natorp adresse aux Recherches logiques dans le chapitre XI de son ouvrage Allgemeine Psychologie de $1912^{77}$ et dans sa recension du premier livre des Idées directrices ${ }^{78}$. Le thème commun à ces deux articles est la fameuse question de la Gegebenheit, du donné ou de ce que Husserl appelle tout simplement en 1901 les phénomènes. Le reproche que Husserl adresse à Natorp dans les $\mathbb{S} \mathbb{8}$ et 16 de la cinquième Recherche est, d'une part, de ne pas distinguer clairement ces contenus «réels» des contenus intentionnels, et, d'autre part, les contenus de couleur - par exemple - des propriétés des objets colorés. Le même genre de reproche est adressé à Brentano dans l'appendice aux Recherches logiques ${ }^{79}$, de même qu'au phénoménisme qu'il attribue aussi bien à l'empirisme britannique qu'à E. Mach dont il est question dans la contribution de Courtine à ce numéro. L'étude de Pradelle porte sur un thème apparenté au précédent, puisque la notion d'intuition dans les Recherches logiques est intimement liée à celle des contenus phénoménaux auxquels elle donne accès de manière immédiate et directe, et ce, par opposition aux actes qui entretiennent avec leurs objets une relation intentionnelle dont les contenus sont conceptuels. Pradelle s'intéresse quant à lui à l'extension de la notion d'intuition aux catégories formelles dans la sixième Recherche et se demande si cet élargissement est justifié. Elle ne l'est pas entièrement dans les Recherches logiques si l'on admet ce que Pradelle appelle le principe de régionalisation de l'intuition, suivant lequel les modalités (ontologiques) qui sont propres à la région sensible sont différentes de celles qui appartiennent au catégorial.

\section{VII}

Revenons maintenant à la disputatio sur l'ouvrage de Smith. Les deux grandes questions qui font l'objet de ces discussions portent sur la nature du noème, notion qui désigne les contenus intentionnels dans le premier livre des Idées directrices, ainsi que sur le sens de la phénoménologie husserlienne dans les Recherches logiques et dans l'ensemble de l'œuvre de Husserl. Les discussions autour de l'ouvrage de D. W. Smith nous donnent l'occasion de soulever la question de savoir ce qu'il advient de la philosophie développée par Husserl durant la période de Halle après les Recherches logiques. Cette question n'est pas nouvelle, elle a été soulevée dès le début des années 1910

76. P. Natorp, Einleitung in die Psychologie nach kritischer Methode.

77. P. Natorp, Allgemeine Psychologie nach kritischer Methode, Buch I.

78. P. Natorp, "Husserls Ideen zu einer reinen Phänomenologie».

79. Sur la critique que fait Husserl de la perception interne de Brentano, cf. D. Fisette «Husserl and Brentano on Descriptive Psychology and Natural Sciences ». 
par les premiers étudiants de Husserl appelés aussi les phénoménologues du Cercle de Munich, lesquels n'ont pas manqué de remarquer dans l'enseignement du maitre les écarts de plus en plus marqués par rapport à la doctrine de l'ouvrage séminal de la phénoménologie. Et en effet, dès après la parution des Recherches logiques, Husserl conteste le sens même de la phénoménologie telle que définie dans cet ouvrage, c'est-à-dire de la phénoménologie en tant que psychologie descriptive, et de nombreux lecteurs de Husserl y ont vu un tournant radical de sa pensée et une réorientation entière de son projet philosophique dans le sens de la tradition kantienne, sinon de l'idéalisme transcendantal. Une des thèses centrales que défend Smith dans son ouvrage et qu'il oppose explicitement à la lecture «discontinuiste» de la philosophie de Husserl entre la période de Halle et celle de Göttingen (1901-1916) est qu'il existe une certaine continuité dans le développement intellectuel de Husserl et dans le projet philosophique qu'il met en place dans le premier volume des Recherches logiques, et que les modifications qu'il fait subir à sa phénoménologie tant sur le plan méthodologique que conceptuel visent en fait un approfondissement et une extension du projet initial ${ }^{80}$. La thèse de la continuité est doublée d'une autre thèse qui est abondamment discutée dans cette disputatio, à savoir la thèse de la systématicité suivant laquelle:

Husserl's diverse theories in logic, ontology, phenomenology, and epistemology are bound together as parts of a single, wide-ranging philosophical theory of meaning, language, essence (species, form, number), part-whole, consciousness (intentionality), and knowledge. [...] The partial theories are parts of the grand theory, which is Husserl's philosophical system ${ }^{81}$.

Ce projet philosophique déjà esquissé dans les Prolégomènes n'est pas étranger à ce qu'il appelle après Bolzano une Wissenschaftslehre ou théorie de la science. Smith soutient que c'est ce «système" philosophique qui assure non seulement l'unité des Recherches logiques mais encore la continuité et le caractère systématique du projet philosophique de Husserl dans son ensemble.

Ces deux thèses audacieuses de Smith sont bien documentées dans son ouvrage, et elles sont le fruit d'une longue maturation qui remonte à ses travaux du début des années 1980 dans un ouvrage désormais classique publié avec R. McIntyre: Husserl et l'intentionnalité. Une étude de l'esprit, de la signification et du langage ${ }^{82}$. Dans cet ouvrage, l'étudiant de Føllesdal élabore de manière systématique l'interprétation sémantique de la théorie husserlienne de l'intentionnalité en concevant les noèmes ou contenus inten-

80. Pour une défense convaincante et bien documentée de la thèse de la continuité (entre la période de Halle et celle de Göttingen), cf. l'ouvrage récent de J. N. Mohanty The Philosophy of Edmund Husserl. A Historical Development.

81. D. W. Smith, Husserl, p. 69.

82. D. W. Smith \& R. McIntyre, Husserl and intentionality. A study of mind, meaning, and language. 
tionnels sur le modèle du Sinn de Frege, c'est-à-dire comme des entités idéales dont la fonction est de médiatiser la référence des actes à leurs objets. C'est ce qu'on a appelé la lecture frégéenne de la phénoménologie ou l'interprétation de la Côte Ouest américaine qui est au foyer de la discussion de Smith avec J. J. Drummond et E. Marbach. Comme on le sait, ce débat autour du noème n'est pas nouveau, et la littérature sur ce thème est très abondante ${ }^{83}$. Cependant, cette disputatio donne une dimension nouvelle à ce débat en insistant davantage sur la portée philosophique des différentes positions sur cette question et leurs conséquences sur notre compréhension de la philosophe husserlienne ${ }^{84}$. Dans les réponses à ses critiques, Smith reconnaît que la conception qu'il se fait de la philosophie de Husserl dans son ensemble repose en bonne partie sur son interprétation de sa théorie de l'intentionnalité:

On connaît bien le dictum de Dagfinn Føllesdal selon lequel la théorie du noème élargit la théorie de la signification du langage vers toutes les expériences intentionnelles en général. On voit ici cette idée centrale ramifiée dans le système husserlien!

En revanche, Marbach et surtout Drummond, qui a publié plusieurs ouvrages critiques contre cette interprétation sémantique de l'intentionnalité chez Husserl ${ }^{85}$, interprète le noème comme un objet visé ou l'objet entre parenthèses parce que le noème n'est accessible dans cette perspective qu'à partir d'une certaine attitude, dite transcendantale, laquelle n'est elle-même possible que grâce à cet artifice méthodologique appelé la réduction phénoménologique. D'où la conception que se font les défenseurs du modèle perspectiviste de la phénoménologie de Husserl après les Recherches logiques ${ }^{86}$.

Un des enjeux de ce débat concerne la place qui revient à la phénoménologie dans le programme philosophique que Smith attribue à Husserl. Car Smith distingue le projet philosophique de Husserl en tant que philosophie

83. Cf. J. J. Drummond, Husserlian Intentionality and Non-Foundational Realism: Noema and Object et D. Fisette, Lecture frégéenne de la phénoménologie.

84. Cf. Zahavi, Husserl's Phenomenology (Stanford, Stanford University Press, 2003), p. 58. Dans sa critique de l'interprétation de la Côte Ouest, Zahavi va même jusqu'à dire que «any attempt to support this narrow meaning-theoretical, semantic interpretation by refering to the places where Husserl speaks explicitly about the constitution of sense is useless, since this maneuver overlooks that Husserl has transcended the objectivistic distinction between meaning and being through his effectuation of the transcendental réduction » (p. 63).

85. Cf. entre autres J. J. Drummond, op. cit.

86. La référence des défenseurs de ce modèle est $\mathrm{R}$. Sokolowski, dont les positions sur la philosophie de Husserl sont clairement exposées dans son ouvrage Introduction to Phenomenology. Un autre défenseur du modèle perspectiviste est $\mathrm{D}$. Zahavi qui, dans son ouvrage sur la phénoménologie de Husserl, interprète le tournant transcendantal durant cette période dans le sens d'une conversion à une forme d'idéalisme: «After Husserl's transcendental turn, idealism is understood as a position that defends the transcendental primacy of subjectivity, a primacy that Husserl considers to be so central to phenomenology that he even identifies phenomenology and transcendental idealism» (Husserl's Phenomenology, p. 69). 
première de la phénoménologie qu'il conçoit comme «l'étude de la conscience telle qu'on en fait l'expérience du point de vue de la première personne ${ }^{87}$ ", dans laquelle sa théorie de l'intentionnalité occupe une place centrale. Drummond rejette cette distinction en faisant valoir qu'elle revient à confiner la phénoménologie au rôle auxiliaire de science régionale au même titre que l'ontologie et la logique. Selon lui, cette conception étroite de la phénoménologie correspond à la définition des Recherches logiques comme psychologie descriptive en tant que science des phénomènes psychologiques, conception que Husserl aurait abandonnée quelques années plus tard au profit de la phénoménologie transcendantale. Drummond conteste donc et la thèse de la continuité (la phénoménologie comme psychologie descriptive est "pré-phénoménologique ») et en partie la thèse de la systématicité en faisant valoir que la phénoménologie transcendantale transcende les sciences régionales et qu'elle est fondatrice de l'ontologie, de la logique et de la théorie de la connaissance. Elle est donc philosophie première au sens traditionnel du terme.

Dans la deuxième partie de son commentaire, Drummond cherche à montrer que le noème doit être compris à la lumière de la phénoménologie comprise comme philosophie transcendantale, c'est-à-dire en tant qu'objet réduit ou entre guillemets, et appréhendé transcendentalement depuis l'attitude phénoménologique ${ }^{88}$. Il conteste la relation étroite que Smith établit entre la phénoménologie et l'ontologie, relation qui se traduit dans sa conception du noème en tant que «moment» dépendant d'un acte, conception que je ne commenterai pas ici. Le commentaire de Marbach s'appuie sur les mêmes prémisses de base (celles de Sokolowski) et l'auteur se livre à un exercice herméneutique visant à corriger l'interprétation que Smith fait des sections pertinentes des Idées directrices, dans lesquelles Husserl expose la nouvelle version de sa théorie de l'intentionnalité et sa conception du noème. Il en a plus particulièrement contre la fonction médiatrice que le modèle californien assigne au noème. Contrairement aux modèles concurrents $^{89}$, le modèle médiateur conçoit l'intentionnalité comme une relation à trois places entre l'acte, le noème et l'objet, et distingue donc le noème à la fois de l'objet simpliciter et de l'état psychique dont il est le contenu. Le noème est compris ici comme signification, ou Sinn, comme une entité idéale que l'on peut interpréter dans le sens bolzanien des propositions en soi et des

87. Smith, Husserl, p. 188.

88. Smith (Husserl, p. 309-310) reformule clairement cette conception du noème: «where an act $\mathrm{A}$ intends an object $\mathrm{X}$ as having a feature $\mathrm{F} 1,(1)$ the object of the act is $\mathrm{X}$, and the noema of the act is X-as-F1, where $\mathrm{X}$ may or may not exist and $\mathrm{X}$ may or may not really be $\mathrm{F} 1$, and (2) $\mathrm{X}$ is a pole of identity in the system $\{\mathrm{X}$-as-F1, X-as-F2, Xas-F3, . . \}, where Fi are purported features of $\mathrm{X}$ as intended in alternative acts directed toward $\mathrm{X}$ from different perspectives."

89. En ce qui concerne les modèles concurrents, cf. l'introduction à The Cambridge Companion to Husserl. 
Idées platoniciennes selon l'interprétation qu'en fait Lotze et dont il a été question précédemment. Mais Marbach soutient que cette interprétation du noème ne cadre pas avec le transcendantalisme de Husserl. Smith résume de manière élégante l'argumentation de Marbach contre le modèle médiateur sur l'exemple de la perception d'un arbre:

Le noème en corrélation avec un acte de conscience est l'objet en tant que visé dans l'acte.

Il n'y a qu'un objet considéré, l'objet simpliciter, l'arbre lui-même (si un tel arbre existe);

Cet objet peut être $v u$ de deux manières différentes: comme il est dans la nature (l'arbre simpliciter) et comme il est en tant que visé (qu'il soit ou non tel qu'il est visé ou qu'une telle chose existe ou non);

Ainsi, il n'y a pas un autre objet qui est l'objet-en-tant-que-visé: il n'y a pas deux objets, mais simplement deux façons de voir le seul et même objet;

Conséquemment, le noème n'est pas une entité qui médiatise la relation intentionnelle d'un acte vers un objet: il n'y a pas de troisième entité impliquée dans la relation acte-objet.

Des trois commentaires sur l'ouvrage de Smith, celui de J.-M. Roy est sans doute le plus sympathique à l'approche de Smith en ce qu'il reconnaît le bien-fondé d'une approche sémantique non seulement de l'intentionnalité, mais aussi de la phénoménologie husserlienne dans son ensemble. Il insiste plus particulièrement sur l'appartenance de Husserl à la tradition sémantique qu'il conçoit après Dummett depuis la thèse que la logique philosophique est philosophie première, avec cette réserve toutefois - laquelle fait écho à la critique que Husserl adresse à Bolzano dans les Prolégomènes — que la logique philosophique ne saurait faire l'économie de la théorie de la connaissance ou simplement la remplacer. Roy soutient que la sémantique husserlienne dans les Recherches logiques présente des avantages par rapport à celles de Frege et de Russell sur la question du fondement de la logique.

En explicitant l'arrière-plan théorique des recherches de Husserl durant la période de Halle, nous avons proposé un aperçu de la contribution des articles réunis dans ce numéro de Philosophiques à notre connaissance de la philosophie du jeune Husserl. Bien que ces études portent toutes sur des aspects essentiels des recherches de Husserl durant cette période et traitent des étapes essentielles qui ont marqué l'évolution de sa pensée jusque dans les Recherches logiques, elles n'épuisent bien évidemment pas l'ensemble des ressources de ce que qu'on appelle parfois la période pré-phénoménologique dans la philosophie husserlienne. Pensons par exemple aux Recherches logiques, que d'aucuns considèrent comme l'œuvre maîtresse de la phénoménologie, mais qui, comme le montre notamment la disputatio autour de l'ouvrage de Smith, est un ouvrage dont l'interprétation demeure à ce jour encore problématique en dépit des nombreux travaux qui lui ont été 
consacrés $^{90}$. Néanmoins, il ressort clairement de ces études que l'ouvrage séminal de la phénoménologie est le résultat de recherches entreprises dès son arrivée à Halle et que les grands principes qui gouvernent les recherches de cet ouvrage sont déterminants pour le développement ultérieur de sa philosophie jusque dans ses derniers travaux à Freiburg. La thèse continuiste veut en effet que la valeur des différentes versions de la phénoménologie que Husserl a élaborées après son départ de Halle se mesure d'abord et avant tout à ces principes. Contester cette thèse de la continuité au nom d'un prétendu tournant idéaliste dans le sens de la philosophie postkantienne, sinon néokantienne, c'est se méprendre sur le sens des préoccupations philosophiques de Husserl et les problématiques qui l'ont toujours motivé. Â cet égard, une meilleure connaissance de l'arrière-plan historique de la période de Halle et des problématiques qui étaient au cœur des préoccupations des philosophes et scientifiques durant la deuxième moitié du XIX ${ }^{e}$ jusqu'au début des années 1930 permettent de mieux comprendre les tenants et aboutissants de la phénoménologie husserlienne. Ces problématiques ont bien un rapport avec l'école de Brentano et la tradition autrichienne en philosophie dans la mesure où, en réaction à la philosophie postkantienne, tous les philosophes qui se sont réclamés de ces courants de pensée étaient en quête d'une philosophie en tant que science rigoureuse; mais elles en sont distinctes. Les études réunies dans cet ouvrage donnent une idée de la diversité de ces problématiques et des interlocuteurs privilégiés de Husserl durant la période de Halle. Puissent-elles susciter l'intérêt des philosophes pour un des segments les plus riches de l'histoire de leur discipline.

\section{Références}

Antonelli, M. Seiendes, Bewusstsein, Intentionalität im Frühwerk von Franz Brentano, Freiburg-München, Alber, 2001.

Ash, M. G. Gestalt Psychology in German Culture 1890-1967, Cambridge, Cambridge University Press, 1995.

Atten, M. van. Brouwer meets Husserl. On the Phenomenology of Choice Sequences, Berlin, Springer, 2007.

Bolzano, B. 1837. Wissenschaftslehre. 4 vol., Sulzbach, Seidel.

Brentano, F. Psychologie vom empirischen Standpunkte, Leipzig, Duncker \& Humblot, 1874.

—. Briefe an Carl Stumpf 1867-1917, G. Oberkofler (dir.), Graz, Akademische Druck- und Verlaganstalt, 1989.

Capek, K. Gespräche mit Masaryk, Mindelheim, Sachon Verlag. 1990.

Centrone, S. Logic and Philosophy of Mathematics in the Early Husserl, Berlin, Springer, 2010.

90. Plusieurs ouvrages ont été récemment consacrés aux Recherches logiques (19001901) à l'occasion du centième anniversaire de sa parution. Cf. entre autres ouvrages ceux que nous avons publiés: Aux origines de la phénoménologie et Husserl's Logical Investigations Reconsidered. 
Drummond, J. J. Husserlian Intentionality and Non-Foundational Realism: Noema and Object. Dordrecht et Boston, Kluwer Academic Publishers, maintenant Springer, 1990.

Ehrenfels, C. von. 1890. "Uber Gestaltqualitäten». In Vierteljahrsschrift für wissenschaftliche Philosophie, vol. 14. Trad. fr. D. Fisette, in D. Fisette et G. Fréchette (dir.), À l'école de Brentano. De Würzburg à Vienne, Paris, Vrin, 2007, p. 227-262.

Fabian, R. "Leben und Wirken von Christian von Ehrenfels: Ein Beitrag zur intellektuellen Biographie», in R. Fabian (dir.), Christian von Ehrenfels: Leben und Werk. Amsterdam, Rodopi, 1986.

Fisette, D. Lecture frégéenne de la phénoménologie, Paris, Éclat, 1994.

- (dir.), Husserl's Logical Investigations Reconsidered. Dordrecht, Kluwer, 2003.

-. (dir.), Aux origines de la phénoménologie, Paris, Vrin, 2003.

—. "La philosophie de Stumpf, ses origines et sa postérité", in Carl Stumpf. Renaissance de la philosophie, Paris, Vrin, 2006, p. 11-112.

-. (dir.), Carl Stumpf. Renaissance de la philosophie, Paris, Vrin, 2006.

—. Avec G. Fréchette (dir.), À l'école de Brentano. De Würzburg à Vienne, Paris, Vrin, 2007.

—. "Stumpf and Husserl on Phenomenology and Descriptive Pychology ", Gestalt Theory, vol. 32, n 2, 2009, p. 115-128.

—. "Husserl and Brentano on Descriptive Psychology and Natural Sciences", U. Melle (dir.), Husserl, Phenomenology and the Sciences, Berlin, Springer (à paraître).

—. "Husserl et Lotze», Dialogue (à paraître).

—. "Le legs de Brentano", D. Fisette, G. Fréchette (dir.), À l'école de Brentano. De Würzburg à Vienne, Paris, Vrin, 2007, p. 7-160.

Fréchette, G. Gegenstandslose Vorstellungen. Bolzano und seine Kritiker, Academia, Sankt-Augustin, 2009.

Frege, G. «Rezension von: E. Husserl, Philosophie der Arithmetik I», Zeitschrift für Philosophie und philosophische Kritik, vol. 103, 1894, p. 313-332.

Gerlach, H.-M. et H. R. Sepp (dir.). Husserl in Halle. Daedalus, vol. 5, Francfurt, Peter Lang, 1994.

Husserl, E. Über den Begriff der Zahl (Psychologische Analysen), Halle, Heynemann'sche Buchdruckerei, 1887.

- Husserliana III/1, Ideen zu einer reinen Phänomenologie und phänomenologischen Philosophie, 1. Buch: Allgemeine Einführung in die reine Phänomenologie, K. Schuhmann (dir.), La Haye, Nijhoff, 1976.

—. Husserliana XII, Philosophie der Arithmetik. Lothar Eley (dir.), La Haye, Nijhoff, 1970.

- Husserliana XVII, Formale and transzendentale Logik, P. Janssen (dir.), La Haye, Nijhoff, 1974.

- Husserliana XIX/1: Logische Untersuchungen. Zweiter Band, 1. Teil. Untersuchung zur Phänomenologie und Theorie der Erkenntnis, La Haye, Nijhoff, 1984.

- Husserliana XIX/2: Logische Untersuchungen. Zweiter Band, 2. Teil. Untersuchung zur Phänomenologie und Theorie der Erkenntnis, La Haye, Martinus Nijhoff, 1984. 
-. Husserliana XXI, Studien zur Arithmetik und Geometrie, E. Strohmeyer (dir.), La Haye, Nijhoff, 1983.

—. Husserliana XXII, Aufsätze und Rezensionen (1890-1910), B. Rang (dir.), La Haye, Nijhoff, 1979.

- Husserliana XXVII, Aufsätze und Vorträge. 1922-1937, H. R. Sepp (dir.), Dordrecht, Kluwer, 1988.

- Husserliana XXIX, Die Krisis der europaïschen Wissenschaften und die transzendentale Phänomenologie. Ergänzungsband. Texte aus dem Nachlass, R. N. Smid (dir.), Dordrecht, Kluwer, 1992.

- Husserliana XL: Untersuchungen zur Urteilstheorie: Texte aus dem Nachlass (1893-1918), R. Rollinger (dir.), Dordrecht, Springer, 2009.

-. Briefwechsel. Die Brentanoschule, Bd. I, E. Schuhmann \& K. Schuhmann (dir.), Berlin, Springer, 1994.

—. Briedwechsel. Die Göttinger Schule, Bd. III, E. Schuhmann \& K. Schuhmann (dir.), Berlin, Springer, 1994.

-. Briefwechsel . Die Neukantianer, Husserliana Dokumente, Bd. V, E. Schuhmann \& K. Schuhmann (dir.), Berlin, Springer, 1994.

—. Briedwechsel. Institutionelle Schreiben, Bd. VIII, E. Schuhmann \& K. Schuhmann (dir.), Berlin, Springer, 1994.

—. Husserliana, Materialien Bd, 1, Logik. Vorlesung 1896, E. Schuhmann (dir.), Dordrecht, Kluwer Academic Publishers, 2001.

—. «Ursprüngliche Druckfassung der Abhandlung "Intentionale Gegenstände" von Husserl», in Karl Schuhmann, «Husserls Abhandlung "intentionale Gegenstände”. Edition der ursprünglichen Druckfassung », in Brentano Studien, III, 1990/91, pp. 142-176.

_. "Vorlesung über den Begriff der Zahl (WS 1889-1890) », The New Yearbook for Phenomenology and Phenomenological Philosophy, vol. V, 2005, p. 278308.

—. «Erinnerungen an Franz Brentano », O. Kraus (dir.), Franz Brentano. Zur Kenntnis seines Lebens und seiner Lehre, Munich, Beck, 1919, p. 151-167; trad. fr. R. Brisart, "Souvenirs de Franz Brentano », in D. Fisette et G. Fréchette (dir.), À l'école de Brentano. De Würzburg à Vienne, Paris, Vrin, 2007, p. 163-173.

_. "Entwurf einer Vorrede zu den Logischen Untersuchungen ", Tijdschrift voor Filosofie, vol. I, p. 106-133, 319-339.

Husserl, Malvine. «Skizze eines Lenbenbildes von E. Husserl», K. Schuhmann (dir.), Husserl Studies, vol. 5, 1988, p. 105-125.

Künne, W. «Husserl and Bolzano on Questions », in D. Fisette (dir.), Husserl's Logical Investigations Reconsidered. Dordrecht, Kluwer, 2003.

Lotze, H. Logik. Drei Bücher vom Denken, vom Untersuchen und vom Erkennen, $2^{\mathrm{e}}$ éd., G. Misch (dir.), Leipzig, Meiner, 1912.

Mach, E. Beiträge zur Analyse der Empfindungen, $1^{\mathrm{e}}$ éd., Jena, Gustav Fischer, 1886.

—. «Bemerkungen zur Lehre vom räumlichen Sehen », Zeitschrift für Philosophie und philosophische Kritik, neue Folge, vol. 46, 1865, p. 1-5.

Mulligan, K. \& B. Smith. "Mach and Ehrenfels: The Foundations of Gestalt Theory ", in Smith (dir.), 1988, p. 124-157.

Meinong. Über Annahmen, Gesamtausgabe Bd. IV, R. Haller (dir.), Graz, Akademische Druck- und Verlaganstalt, 1977. 
Mohanty, J. N. The Philosophy of Edmund Husserl. A Historical Development, New Haven, Yale University Press, 2008.

Natorp, P. Einleitung in die Psychologie nach kritischer Methode, Freiburg, Mohr, 1888.

- Allgemeine Psychologie nach kritischer Methode, 1. Buch: Objekt und Methode der Psychologie, Tübingen, Mohr Siebeck, 1912.

- - "Husserls Ideen zu einer reinen Phänomenologie», Logos, vol. VII, 19171918, p. 224-246.

—. "Zur Frage der logischen Methode. Mit Beziehung auf Edmund Husserls Prolegomena zur reinen Logik», Kant Studien, vol. 6, 1901, p. 270-283.

Novac, J. (dir.). On Masaryk, Amsterdam, Rodopi, 1988.

Palágyi, M. Der Streit der Psychologisten und Formalisten in der modernen Logik, Leipzig, 1902.

Peckhaus, V. (dir.), Oskar Becker und die Philosophie der Mathematik, München, Wilhelm Fink Verlag, 2005.

Rollinger, R. D. Husserl's Position in the School of Brentano, Dordrecht, Kluwer, 1999.

Schuhmann, K. Husserl-Chronik (Denk-und Lebensweg Edmund Husserls). Husserliana Dokumente I, La Haye, Nijhoff, 1977.

—. (dir.). "Husserls Abhandlung "Intentionale Gegenstände". Edition der ursprünglichen Druckfassung ", Brentano Studien 3, 1990-1991, p. 137-176.

—. "Husserls Abhandlung "Intentionale Gegenstände”. Edition der ursprünglichen Druckfassung ", in Brentano Studien, III, 1990, p. 137-176.

- "Stumpfs Vorstellungsbegriff in seiner Hallenser Zeit", Brentano Studien 9 (2000/2001), 63-88 «Husserls Manuskripte zu seinem Doppelvortrag von $1901 »$, in Husserl Studies, vol. 17, 2001, p. 87-123.

-. "Husserl and Masaryk», in J. Novac (dir.), 1988, p. 129-156.

—. "Stumpfs Vorstellungsbegriff in seiner Hallenser Zeit», Brentano Studien, vol. 9, 2000-2001, p. 63-88.

Smith, B. (dir.). Foundations of Gestalt Theory, Munich, Philosophia, 1988.

—. "Gestalt Theory: An Essay in Philosophy ». In Foundations of Gestalt Theory, Barry Smith (dir.), Munich, Philosophia, 1988, p. 11-80.

Smith, B. \& D. W. Smith (dir.). The Cambridge Companion to Husserl, Cambridge, Cambridge University Press, 1995.

Smith, D. W. "The Unity of Husserl's Logical Investigations: then and now", D. Fisette (dir.) Husserl's Logical Investigations Reconsidered, Dordrecht, Kluwer, 2003, p. 21-34.

-. Husserl, Londres et New York, Routledge, 2007.

Smith, D. W. \& R. McIntyre. Husserl and intentionality. A study of mind, meaning, and language, Dordrecht, Reidel, 1982.

Sokolowski, R. Introduction to Phenomenology (Cambridge, Cambridge University Press, 2000).

Stumpf, C. Über den psychologischen Ursprung der Raumvorstellung, Leibzig, S. Hirzel, 1873.

- " "Autobiographie», trad. fr. D. Fisette, Carl Stumpf. Renaissance de la philosophie, D. Fisette (dir.), Paris, Vrin, 2006, p. 255-307.

- "Rezension von "Ernst Mach, Beiträge zur Analyse der Empfindungen" ", Deutsche Literaturzeitung, vol. 27, 1886, p. 947-948. 
—. "Psychologie und Erkenntnistheorie ", Abhandlungen der Königlich Bayerischen Akademie der Wissenschaften, vol. 19, 2nd part, München, Franz, 1891, p. 465-516.

Tieszen, R. Phenomenology, Logic, and the Philosophy of Mathematics, Cambridge, Cambridge University Press, 2005.

Twardowski, K. Zur Lehre vom Inhalt und Gegenstand der Vorstellungen. Eine psychologische Studie, Vienna, Hölder, 1894.

Zahavi, D. Husserl's Phenomenology, Stanford, Stanford University Press, 2003. 